\title{
Lecture d'une pratique de coaching : un détour par la pensée chinoise
}

Dominique Steiler

\section{(2) OpenEdition}

1 Journals

Édition électronique

URL : http://journals.openedition.org/communicationorganisation/3346

DOI : 10.4000/communicationorganisation.3346

ISSN : $1775-3546$

Éditeur

Presses universitaires de Bordeaux

Édition imprimée

Date de publication : 1 janvier 2006

Pagination : 118-135

ISSN : 1168-5549

\section{Référence électronique}

Dominique Steiler, «Lecture d'une pratique de coaching : un détour par la pensée chinoise »,

Communication et organisation [En ligne], 28 | 2006, mis en ligne le 19 juin 2012, consulté le 19 avril

2019. URL : http://journals.openedition.org/communicationorganisation/3346 ; DOI : 10.4000/

communicationorganisation.3346

Ce document a été généré automatiquement le 19 avril 2019

(C) Presses universitaires de Bordeaux 


\title{
Lecture d'une pratique de coaching : un détour par la pensée chinoise
}

\author{
Dominique Steiler
}

1 "Qu'est-ce qu'une intervention de coaching "? C'est à partir de cette question, parfois des émotions qu'elle suscite et de la difficulté d'y répondre simplement, que nous avons décidé de nous interroger et d'éclairer notre pratique de coaching; le terme "d'accompagnement» nous semblant d'ailleurs plus approprié. Pour la repérer dans le dédale (délirant) « des coaching », nous la positionnerons à la croisée du développement personnel et du développement managérial. C'est à partir de l'expérience de terrain que nous avons choisi de réfléchir, considérant, comme le soulignait André Gide, ${ }^{1}$ que " toute connaissance que n'a pas précédé une sensation (nous) est inutile ».

2 Il est important de souligner que notre intention n'est pas de nous opposer aux autres pratiques, mais bien plus de partager avec elles afin de susciter les échanges. Pour cela, nous déroulerons les idées sur un chemin en quatre étapes. C'est la narration d'une "histoire de coaching" qui servira d'introduction et de présentation en soulevant les éléments clés. Les différences principales avec des approches plus conventionnelles permettront ensuite de visualiser les spécificités, mais aussi les doutes de cette pratique. Ces derniers sont certainement les moteurs qui nous ont conduits à réfléchir et à lire notre action à travers un regard à « double foyer ». La science du concret et sa métaphore du bricoleur, issues des travaux de Claude Lévi-Strauss, permettront une première lecture décalée. Le passage par la Chine n'aura ensuite ni vocation exotique ou marketing de création ou de justification d'une nouvelle pratique, ni ambition de chercher de nouvelles solutions à nos problèmes. Avec respect pour la pensée chinoise et en regard de nos connaissances des pratiques occidentales, il aura pour ambition de nous donner une possibilité de penser "hors cadre». Il permettra une lecture de notre pratique à travers une culture totalement différente de la nôtre et généralisée depuis plus de 2500 ans. Ces détours nous conduiront à questionner enfin les fondements d'une intervention de coaching, dans sa démarche, ses contenus et son efficacité.

3 Un dernier point nous semble important: préciser notre positionnement face aux différents points exposés dans ce travail. Chacun d'entre eux, à des fins de 
compréhension, représente une certaine amplification de leur réalité, une vision pure qui finalement n'existe jamais réellement en tant que telle. Il s'agit bien là de présenter des modèles généraux, issus d'archétypes, pour tenter de comprendre une réalité à visages multiples.

\section{Histoire de Coaching}

4 «Une connaissance commune m’a parlé de vos interventions. Je suis intéressé et je voudrais voir avec vous si nous pouvons envisager une action de coaching »- c'est ainsi que démarre le premier contact avec le P.D.G. d'une société de 400 personnes du secteur industriel (Marc - M). À l'issue d'un court entretien téléphonique, nous convenons d'une première rencontre dont l'objet est une présentation mutuelle destinée à partager notre vision respective d'une démarche de coaching.

En début de rencontre, Marc m'avoue être intrigué par la méthode de biofeedback ${ }^{2}$ que j'utilise. Après une courte présentation de son activité professionnelle et de son environnement, il aborde sa demande principale qui porte sur un problème de gestion du stress qu'il veut régler pour améliorer sa performance. Le peu qu'il connaît du biofeedback lui semble être prometteur et il souhaite que l'on utilise cet outil.

Le texte qui va suivre présente des extraits de cette première rencontre.

7 Dominique (D) - Votre demande entre bien dans le cadre d'une démarche de coaching, située entre un développement personnel et un développement managérial. Peut-être que le premier point sur lequel je tiens à échanger avec vous est celui de cette notion de performance que vous incluez dans votre demande. Dans cette démarche de coaching, la performance ne sera pas considérée comme un objectif à atteindre. Elle conservera sa place, celle d'un but, plutôt lointain, que l'on désire atteindre sans complètement connaître sa forme finale. Ce qui nous intéressera plus, ce sera donc les objectifs que nous dessinerons au fur et à mesure de nos rencontres, qui seront eux les éléments à maittriser pour atteindre cette performance. Autrement dit, nous distinguerons deux temps dans la vie professionnelle: le temps de l'apprendre et le temps de l'efficacité. Avancer dans l'apprendre, comprendre la situation et lui donner une voix nous permettra de construire les conditions à partir desquelles la performance découlera.

M - Est-ce que cela veut dire que notre contrat ne définira pas d'objectifs spécifiques, ni de plan précis?

D - Si, mais d'une manière spécifique! Il définira ce que vous ciblez aujourd'hui comme étant votre demande pour s'en servir de point de repère. Il précisera ensuite, mais sans les pré-planifier de façon rigide, les étapes, les méthodes et outils et l'objet final de l'intervention. Ce qui importera, c'est bien plus nous adapter en continu aux contextes et aux situations, pour en construire une compréhension commune à partir de nos échanges. C'est à partir de cette compréhension que nous construirons la démarche à suivre.

$10 \mathrm{M}$ - Je pensais que nous utiliserions assez rapidement le biofeedback ou d'autres outils ou concepts courants en coaching?

11 D - La plupart des outils et concepts présentés comme des fondamentaux ou des spécificités du coaching sont de bons outils et sont très utiles lorsqu'ils s'appliquent bien aux contextes pour lesquels ils ont été élaborés. Mais il me semble qu'il faut, en premier 
lieu, bien plus comprendre la situation dans laquelle vous vivez, savoir entrer petit à petit dans une attitude d'apprendre, d'ouverture qui enrichisse vos perceptions, vos ressentis, vos comportements et la vision de votre environnement afin, alors, d'utiliser l'outil approprié. De plus, il ne s'agit pas d'entrer dans une approche directe d'utilisation de tel outil pour telle fin. Dans ma façon d'aborder le coaching, les outils sont distillés, ingérés, digérés puis oubliés, en ce sens où ils font alors partie du coach ou de la personne, ils sont devenus des automatismes hors du champ immédiat de la conscience et sous une nouvelle forme plus personnelle. Ils pourront alors être abordés de manière directe.

J'entends par outils, non seulement les instruments concrets dont on se sert, mais également tout concept qui en coaching est utilisé comme un outil de pensée. Prenons ici l'exemple de l'empathie et de son usage courant qui pourrait être la capacité de ressentir les choses comme si j'étais à la place d'autrui. Un premier temps sera d'amener de manière simple le manager à la pratiquer, sans la nommer. Ceci jusqu'au moment où elle ne sera plus empathie, mais empathie de M.A, reconnue comme une habileté propre, distincte de l'empathie de M. Coach. Elle ne sera alors plus uniquement un moyen pour résoudre un problème, mais une habileté qui s'est installée dans une connaissance en action, qu'on laisse parler avec le souci de vivre mieux la situation. Oubliée dans sa forme consciente, mais totalement présente dans son expression immédiate, elle laisse place à l'automatisme, qui tout comme pour le sportif, permet de ne plus penser son geste, mais qui l'inscrit simplement dans le courant des choses. C'est alors qu'elle pourra être dénommée et questionnée par un regard critique, tel qu'a pu le faire par exemple Lacan qui disait : «Si l'empathie c'est se mettre à la place de l'autre, alors où est l'autre?».

Cette seconde phase, par une articulation de la pratique et du concept, par une vue de dessus, conduira finalement à une prise de conscience de la forme qu'a prise pour le manager son appropriation personnelle de l'outil. Elle interviendra donc plus tard dans la démarche: une fois que l'attitude d'apprendre a fait son chemin chez le manager, que certains insights ont eu lieu ou encore comme le souligne Rogers ${ }^{3}$ que le manager face au problème évoqué « en vient à se voir de façon différente... devient plus acceptant... plus ouvert à l'évidence, à la fois pour ce qui se passe à l'extérieur (et) en lui-même ».

$14 \mathrm{M}$ - Je reste pourtant très intéressé par le fait de connaître différentes techniques pour mieux gérer mon stress professionnel et c'est aussi cela que j'attends de vous.

D - Je pense qu'une trop grande focalisation sur la maittrise d'un outil fait courir le risque d'une croyance d'un pouvoir sur les choses. La personne croit alors qu'il existe quelque part un outil efficace à $100 \%$ qui lui permettra de résoudre son problème.

Il me semble plus intéressant dans un premier temps d'expérimenter, de comprendre qu'il existe différents points de vue et différentes approches et que chacune possède sa part de réussite et d'échec. Ce vécu au sens large, intellectuel, émotionnel, physique et comportemental se fait dans une relation de coaching moins pour l'autre qu'auprès de l'autre et dans l'échange avec lui. Une certaine vision du coaching parle d'une démarche en pouvoir pour ${ }^{4}$, qui déjà ne place plus le rapport au même endroit que le pouvoir sur. Pour mon cas, le bon terme serait plus un pouvoir avec, une attitude côte à côte, de partage à travers lequel chacun apporte et chacun est ouvert à l'expérience de l'autre. Le coach est en pouvoir avec le manager exactement comme le manager est en pouvoir avec le coach. Il n'y a pas là de relation de force, ni à l'autre, ni à l'outil, ni au problème. Il n'y a pas non plus de relation d'autorité, le coach ou l'outil n'ont pas plus autorité que le manager sur le thème qui sera abordé dans la séance. Chacun possède une partie de pouvoir faire ensemble quelque chose. 

direction. Nous ne travaillerons pas sur le ton, le volume, le débit de sa voix, en considérant a priori leur influence sur la communication. Nous chercherons par contre, étape par étape, une compréhension de la situation réelle et ses composantes dont font partie le contenu de l'exposé, lui-même, les autres participants et la représentation qu'il s'en fait, pour en saisir les potentiels et mener le manager à s'inscrire dans leur courant naturel. Il pourra s'agir ainsi moins d'apprendre une nouvelle méthode que de systématiser une habileté déjà présente en lui. dans le contexte et choisie ensemble, en priorité parmi ce que sait déjà faire le manager, que se posera bientôt la question de la présence du coach. Une fois que le manager se sera approprié l'outil, il arrivera bientôt au point de ne plus voir le coach comme un référent dans ce domaine, mais plus comme une personne avec laquelle il pourra échanger. La fin de l'intervention ne sera alors pas tant décidée que vécue. Le coach proposera alors de clore l'intervention. but en passant par l'acquisition d'outils, nous pourrions conclure de ce cas, que la démarche présentée apparaît pour le moins décalée voire, si nous poussions la critique plus avant, proche d'un bricolage au sens courant du terme.

Après une courte présentation des fondamentaux actuels du coaching, qui nous permettra de préciser simplement les différences avec l'approche présentée, c'est justement un passage par le concept peu connu de bricolage qui nous mènera ensuite à considérer les apports que la pensée chinoise peut introduire pour tenter la généralisation d'une telle pratique. 


\section{Les fondamentaux du coaching : quelles différences?}

Dans la première partie, nous avons pu voir la distance existant entre la demande de Marc et la présentation d'un autre mode d'intervention. Cette demande type de coaching identification d'un écart entre une situation actuelle et un idéal souhaité, planification d'une démarche, recherche d'outils optimaux - est proche de l'offre type que l'on peut trouver dans la littérature ou encore dans les propositions des cabinets spécialisés. Puisque l'un des objets de cet article est de porter un regard sur une autre approche du coaching, sans la juger meilleure ou moins efficace, il nous semble important ici d'éclairer les différences qui peuvent exister entre la pratique présentée en première partie et certains fondamentaux standards de ce type d'intervention.

Nous observerons ces différences à travers plusieurs axes: le principe d'efficacité, la relation au client et à l'intervention, les modalités d'action, l'intégration des outils et enfin le processus de fin d'intervention.

Considérons en premier lieu la différence des schémas d'intervention proposés en vue de l'efficacité. Nous pouvons observer que les écrits sur le coaching préconisent une certaine forme de modélisation qui, par un diagnostic, conduira à l'élaboration d'une démarche structurée et planifiée, intégrant des moyens orientés vers le but initialement visé. Ainsi le présentent Winum (2005) ou encore Kampa-Kokesh et Anderson (2001, p. 208) qui stipulent que la démarche est planifiée «en six étapes : construction de la relation, audit, feedback, planification, mise en cuvre et évaluation finale ». Dans la présentation du cas de Marc, l'évaluation initiale n'est pas abordée comme un diagnostic qui permettrait de définir les moyens et de planifier les actions. Son objet est bien plus une évaluation initiale des forces en présence afin d'identifier le potentiel de situation et de comprendre comment le manager pourra s'y inscrire au mieux. Elle évite ainsi, à terme, de bloquer l'évolution d'une demande souvent non aboutie dans les premiers temps de la démarche. Elle facilitera par ailleurs l'intégration des circonstances contextuelles à venir, non prévues et jugées parasites dans une planification, considérées ici comme des variables incontournables de l'efficacité pragmatique de la démarche.

La relation au client considère souvent que le rôle alloué au consultant est «d'aider la personne à atteindre ses objectifs» (Kilburg, 2000, p. 67) et que "le coaching est proposé au bénéfice du client... " (Conference, 2000). Cette relationqui consiste à " aider la personne " (Délivré, 2002, p. 30)peut être interprétée comme un lien d'autorité de fait du coach sur le manager. Il semble seul détenir les moyens nécessaires à l'évolution de son client dans la situation évoquée. Le poids reposera sur ses seules épaules de bien se placer en pouvoir pour et pas en pouvoir sur. Il sera le seul garant de cette attitude. Le cas présenté en première partie parle d'un pouvoir avec et d'une relation d'accompagnement et de partage dans laquelle chacun détient une part d'autorité face à la situation, donc, une partie des moyens qui seront nécessaires pour avancer dans le processus. Et le bénéfice de l'intervention, qu'en est-il alors? Le coach mène-t-il une action désintéressée ? Ce n'est pas notre avis. Il semble important dans une action partagée de coaching d'afficher, dès le démarrage, les bénéfices mutuels envisagés. Si le manager progresse dans son objectif, le coach quant à lui, au-delà de sa rémunération, par son attitude d'apprendre, poursuit son évolution et renforce son expérience. Échanger avec le manager les raisons pour lesquelles l'action lui apparaît intéressante peut permettre au coach de clarifier son 
engagement, de clarifier la relation, mais aussi renforcer la confiance en la co-laboration (travailler ensemble), facteur clé de succès.

31 Le coaching est très souvent présenté comme un moyen d'action qui doit conduire à l'émergence des potentiels de la personne en vue d'une amélioration de sa performance (Gautier \& Vervisch, 2005 ; SFCoach, 2005 ; Whitmore, 2003). Cette affirmation sous-tend plusieurs idées. Premièrement, que par le développement de ses potentiels, le manager pourra agir sur la performance. Deuxièmement, que c'est son action (voir sa pro-action) qui sera la base principale de sa performance. Enfin, que la démarche de coaching a comme objet de donner les moyens d'agir directement sur cette performance. Une approche différente est de considérer que le contenu de la demande du manager est issu plus probablement d'une interaction entre lui et son environnement, une situation ou un contexte. Dans le cas de Marc, il est proposé d'appréhender la situation dans son ensemble, d'en percevoir les aspects multidimensionnels dont il fait lui-même partie. Par cette approche, il pourra alors en comprendre les dynamiques, en détecter les aspects porteurs pour s'y intégrer et identifier comment infléchir les conditions nécessaires à l'effet visé - le but. Cela ne sera donc plus nécessairement son action, directe, qui améliorera une performance sur laquelle il n'a pas entièrement le contrôle, mais plus l'effet combiné de différents facteurs, personnels et situationnels, qu'il aura appris à identifier et à influencer.

Dans la démarche standard de coaching, les outils sont présentés comme les moyens maitrisés par le coach pour aider le manager à atteindre le but fixé à l'issue de l'audit initial. Ainsi, pour Kilburg, le coach "utilise une large variété de méthodes et de techniques... pour aider la personne à atteindre ses objectifs " $(2000$, p. 67). Nous retrouvons là l'idée d'une action volontaire par le moyen d'un outil, jugé optimal, qui transmet des pouvoirs pour agir sur le problème. Cette approche, associée à la croyance de l'identification d'un problème clair et indépendant - par exemple : «c'est un simple problème de conflit »s'accompagne de la conviction qu'il n'existe qu'une solution unique et de la relégation au rebut de toutes les autres options. À l'opposé, croire en l'interrelation entre les choses, détecter et comprendre les facteurs porteurs de la situation, permettra de considérer toutes les facettes d'une solution et de cumuler leur efficacité mutuelle en s'appuyant avant tout sur les habilités déjà présentes chez le client avant de penser à l'acquisition d'une nouvelle méthode. Alors seulement, si une méthode ou un outil sont appréhendés, ils seront d'abord pratiqués pour provoquer la sensation de leur expérience et déclencher la motivation de leur apprentissage.

33 Pour conclure, si le client souhaite une date de fin d'intervention planifiée, sera distinguée la date de fin contractuelle, de la terminaison du processus d'accompagnement - fin de processus. Cette vision, proche de celle de Hévin et Turner (2002, p. 242) qui différencient une fin organisationnelle et une fin psychologique, s'en distingue de trois manières. Tout d'abord, le contrat prévoira la négociation d'une date d'évaluation, plutôt que de fin, à laquelle se posera la question de la poursuite ou non du processus, ce qui nous semble être prioritaire. Ensuite, sera considérée la notion de fin de processus plutôt que de fin psychologique. Ce dernier terme procède davantage d'une vision très individuelle et mentale, alors que l'idée de processus procède d'une vision globale, tant en ce qui concerne le manager que la situation. Enfin, il nous semble que la fin du processus sera plus vécue que décidée. Ce n'est pas une date fixée a priori ou la fin d'apprentissage d'une méthode qui clôturera l'action de coaching. Cette dernière surviendra à travers la prise de conscience partagée de la disparition du rôle de référent du coach, face au problème et 
dans ce processus d'accompagnement, suite à un certain degré d'intégration (le manager a expérimenté et sait utiliser une démarche), d'autonomie (il sait faire seul) et d'originalité (il sait faire à sa façon).

L'observation des fondamentaux du coaching, tels qu'ils sont présentés dans la littérature managériale, fait ressortir certains archétypes de l'action issus de la philosophie occidentale et qui se sont prolongés dans notre culture, nos croyances, tout comme dans les pratiques économiques et organisationnelles, au point de nous apparaître comme une vérité universelle. Ainsi en est-il du principe central de modélisation qui nous conduit, à partir du diagnostic d'un problème, à envisager une solution idéale et ensuite, à travers un plan, à mettre en œuvre des moyens pour qu'existe au final le but défini.

Il conviendra maintenant, par un détour progressif, d'envisager comment la pratique différente présentée en première partie peut se concevoir ou se généraliser à travers deux autres représentations de l'agir.

\section{Du concept de bricolage à la Chine : regard sur un agir spécifique}

Deux chemins vont nous conduire à percevoir différemment la pratique de coaching énoncée en première partie. Pour éviter d'avoir à reconstruire un parallèle trop systématique entre ces approches et cette pratique, nous nous bornerons ici à ne présenter de ces deux axes que les dimensions ou concepts clés en rapport avec cette démarche de coaching.

Le langage courant attache au mot bricolage une définition, plutôt négative, qui présente le travail (peu efficace) d'une personne sans réelles connaissances ni structures. C'est à l'opposé, dans son sens académique, initié par la métaphore du bricolage dans les travaux de Lévi-Strauss, que nous l'utiliserons comme prisme pour apporter une autre vision de cette pratique de coaching.

Pour compléter ce regard, il nous est apparu intéressant de trouver un autre axe d'analyse dont le référentiel soit aussi ancien que la pensée occidentale, tout en étant totalement hors cadre, par son histoire, sa langue, sa culture et ses pratiques. Nous avons donc choisi de poursuivre le décalage, en transitant par la pensée chinoise, pour présenter une autre grille de lecture de notre pratique de coaching.

\section{Le concept de bricolage}

Le concept de bricolage est issu directement des travaux que Claude Lévi-Strauss a présentés dans son ouvrage intitulé La pensée sauvage (1962). Contrairement à la pensée moderne et occidentale, qui se définit comme structurée ou logique et dans laquelle certains auteurs considèrent la pensée sauvage comme prélogique, Lévi-Strauss va considérer qu'elle est simplement d'une logique différente. Bien qu'il n'ait pas précisément défini le concept de bricolage, il le caractérise en premier lieu par une observation exhaustive et un inventaire systématique de tous les éléments du monde environnant. Dans son chapitre premier, il montre comment cette "science du concret" peut construire du sens à la manière du bricoleur.

Il est intéressant de voir que se sont construits, à partir de la métaphore de Lévi-Strauss, des travaux académiques qui apportent au terme bricolage un sens positif. Ainsi, on le 
voit apparaître en sciences de l'éducation (Perrenoud, 1983), en sciences cognitives (BenAri, 1999), dans les travaux sur les organisations (Weick, 1998), tout comme en sciences du management (Baker, Miner, \& Eesley, 2003).

41 C'est à partir des travaux de Lévi-Strauss et des théories managériales que Duymedjian et Rüling (2005) nous présentent les caractéristiques du bricolage et du bricoleur suivant cinq dimensions qui nous permettront de créer du lien avec la pratique de coaching présentée ici.

La première dimension décrit la vision du monde du bricoleur, en y précisant sa place ainsi que le rapport aux êtres et aux choses qu'il peut ou doit avoir. Il n'est nullement question ici de concevoir un monde décomposable, mais bien plus de l'appréhender dans sa complexité et ses interconnexions. L'homme considérera plutôt son action dans un système où tout vaut, mais où tout ne se vaut pas. En effet, si tous les éléments du système ont un intérêt, y compris les circonstances, ils seront pourtant ordonnés. Cette hiérarchie sera cependant le résultat d'une construction immanente élaborée à partir de son propre rapport au monde.

43 La seconde traite de la connaissance du monde. Dans la connaissance encyclopédique de son monde, le bricoleur cherche les connexions entre les choses et comment elles peuvent agir ou se lier entre elles. Il ne cherche pas une modélisation générale du monde pour le prévoir et l'orienter. Son approche des éléments, donc de la situation ou des outils, sera intime, ouverte et faite d'expériences à l'origine de connaissances. Il ne cherche pas un savoir approfondi, mais plus une expérience incorporée qui tôt ou tard trouvera son utilisation.

Dans l'action sur le monde, la personne n'identifie que partiellement le but en considérant que les circonstances à venir demanderont nécessairement des ajustements. Plutôt que de créer un lien indissociable entre une fin déterminée et des moyens optimaux, elle engage un dialogue avec les éléments de son stock, qu'elle détourne éventuellement, afin d'élaborer un assemblage "qui marche». Son action ne sera pas tant guidée par des règles à respecter que par des ajustements continuels pour poursuivre sur la pente engagée.

Le produit de son action, sa réalisation, sera plus de l'ordre d'un dispositif que d'un résultat. Il ressemblera moins aux spécifications d'un plan qu'à un outil ajusté à la demande. Ce dispositif ne ressemblera à aucun autre, sera spécifique à son office et restera modulable pour une évolution future. Bien entendu, totalement issu de la transformation, il sera dépendant de la personne dont l'investissement aura été important.

Enfin, l'identité intègre de fait la personne dans le dispositif produit. Le bricoleur est impliqué de façon importante. Son rôle ne se limite pas à concevoir, mais surtout à utiliser intimement un dispositif qu'il est le seul à mettre en œuvre, au point finalement que maître d'œuvre, maître d'ouvrage et utilisateur deviennent indissociables.

Si cette approche parle bien des notions évoquées dans l'histoire de Marc - évaluation, transformation, action indirecte et vision globale interconnectée - elle reste néanmoins polémique et nous conduit à nous décaler encore d'un cran, pour nous rapprocher d'une culture où ces pratiques semblent faire encore plus de sens. 


\section{Détour par la pensée chinoise}

48 Afin d'appréhender cette pensée, bien plus vaste on s'en doute que les considérations attachées à notre travail, nous nous proposons de présenter dans leurs différences à la pensée occidentale, les caractéristiques les plus à même de donner du sens à notre pratique de coaching. La tâche n'est pas aisée tant la distance à nos préjugés, à nos convictions et certitudes, voire nos références quotidiennes demande un effort important.

49 Parmi celles-ci, il nous a semblé intéressant de traiter les points suivants : la pensée en action, le principe de compréhension du monde, l'évaluation de la situation, l'action, les modalités de l'action et l'efficacité.

Notre point de départ, lointain, concernera donc cette idée de penser en action. Comme le souligne Kamenarovic (2005), si la pensée occidentale à travers Platon et Aristote avait bien comme origine de préciser des règles de vie, elle s'est ensuite donnée comme objet une recherche de la vérité en prenant le principe de ne jamais l'atteindre. Aussi, rapidement, les penseurs et les décideurs n'ont plus été les mêmes personnes. À l'opposé, la Chine a toujours gardé en ligne de mire la volonté de ne pas séparer théorie et pratique, de ne pas rechercher une vérité universelle, mais plutôt de comprendre le concret dans ses variations multiples. Plutôt que d'appréhender le monde par la question du quoi (connaissance de la vérité), la Chine a privilégié la question du comment «mieux vivre sa nature d'homme en harmonie avec le monde »(Cheng, 1997, p. 34). Ainsi, s'est-elle totalement tournée vers la réalité et le traitement pragmatique des choses.

51 À partir de cette approche du monde, la compréhension de la situation vécue par l'homme ne sera pas abordée sur le même modèle. En Occident, la cause, le problème posé, conduira à l'élaboration d'un modèle planifiant le chemin vers un but idéal. Une fois ce futur élaboré théoriquement, il restera à la volonté de mettre en place la pratique pour imposer et faire aboutir le but escompté. Cette dualité théorie-pratique semble ne plus pouvoir être remise en question tant elle fait partie de notre vie. Ainsi en est-il du général qui trace le plan de bataille à suivre, du financier qui trace la courbe de croissance de son indicateur, ou du manager qui planifie l'atteinte de ses objectifs. Mais, suivant Jullien, nous pouvons aussi considérer «(qu') imposer, (c'est) 'placer sur'... comme pour y soumettre de force» $(1996$, p. 17) sans pouvoir ensuite facilement tenir compte des circonstances. En s'appuyant sur la propension, la pensée chinoise propose une alternative. Elle ne présente pas une situation décomposable et isolée, mais un contexte complexe et interconnecté. Elle n'envisage pas de forme définie idéale à atteindre, mais conserve constamment en mémoire, dans le présent de l'action, son but d'amélioration pratique pour qu'une observation des orientations favorables puisse être exploitée. C'est en Chine, à partir du couple situation (telle qu'elle s'actualise) et potentiel (que l'on peut faire jouer en sa faveur) (p. 33), que la personne ajustera son action pour en tirer profit.

L'évaluation prend donc bien son importance dans la démarche vers l'efficacité. Qu'en estil de cet acte initial d'évaluer? Dans sa vision occidentale, l'évaluation des forces en présence, comme il a déjà été évoqué à plusieurs reprises, sert essentiellement la mesure de l'écart avec l'idéal à atteindre afin de dessiner les moyens transcendants à employer. Ce déroulement de l'ordre des étapes prévues, à partir de l'audit initial, semble présenter " un monde en marche fondé sur des concepts immobiles» $(2005$, p. 59) : l'homme peut prévoir son plan puisqu'il sait d'où il part et où il va. En effet, à partir de l'instant de la mesure, le 
but, le plan et les moyens vont être déterminés comme si rien ne bougeait plus. À l'opposé, si nous poursuivons cette citation, la Chine propose une vision "fondée sur la permanence d'un mouvement» (p.59). L'évaluation initiale ne conduit pas l'homme à figer la situation pour en tirer un plan, mais pour en dessiner un diagramme du déroulement, pour en prévoir sa pente, donc l'étape suivante, et y intégrer son action afin de l'infléchir. La circonstance ne sera pas ici considérée comme périphérique ou son action future comme un " concours », mais centrale à la compréhension d'un déroulement.

L'Agir est au cœur de notre vie occidentale. C'est par l'action que l'homme se montre supérieur aux autres êtres vivants de notre planète. Cette action guidée par la volonté vise une maîtrise complète du monde. Pour Kamenarovic (2005, p. 66), " conçue comme un prolongement de la volonté, (elle) reçoit le statut d'intervention sur le monde». Après le plan, l'action vient modifier, de l'extérieur, les conditions de la situation pour les rendre compatibles avec l'idéal défini. De son côté, la Chine ne conçoit pas l'action en dehors d'un déroulement; elle parle d'ailleurs bien plus de transformation que d'action. L'idée n'est donc pas de forger un acte de modification de la situation, mais au contraire de l'accompagner. Dans la relation à ses semblables, «l'homme de bien aide à s'accomplir ce que les autres ont de bon" (Confucius, 1981, p. 99). Au regard de la Chine, comme le souligne Jullien (2005), l'action est vue comme momentanée, locale et renvoie à un sujet unique (même si c'est un groupe). La transformation, elle, est "globale, c'est l'ensemble concerné qui est transformé..., elle s'étend dans la durée... (et) renvoie moins à un sujet qu'elle ne procède discrètement par influence... » (p.56). Ainsi, si l'action est visible et remarquable, les deux sens de ce mot étant bien valorisés en Occident, elle n'en semble pas moins éphémère au regard d'une transformation discrète et indirecte, mais progressive et inéluctable une fois engagée.

Agir, soit, mais comment? Sous quelles modalités? De manière originale, scande l'Occident. L'action se doit non seulement d'être du propre fait d'un homme défini comme son auteur, ce par quoi l'on reconnaît "l'homme d'action ", mais elle se doit aussi d'être vue pour le rendre visible. Dans notre société, si l'homme veut exister, il doit être à l'origine de son acte, créateur sans confusion possible des éléments qui ont conduit à la performance. Il semble alors important, dans une démarche telle que le coaching, d'entrevoir les possibilités de faire émerger les potentiels du manager, car c'est par eux qu'il fera acte original, qu'il pourra se distinguer d'autrui et progresser. Pour la Chine, être original n'a de sens que dans le ponctuel, le passager. Il s'agit au contraire de retrouver en soi ce qui est originel, sa propre part d'universel, ses vertus au sens où la vertu du feu est de brûler (Kamenarovic, 2001), afin de mettre à jour ce en quoi l'on est "bon", ce pour quoi l'on est fait, pour l'inscrire dans la situation et interagir au mieux avec les éléments présents. Ainsi, l'outil ne sera pas appris pour être compris puis appliqué de façon artificielle à une situation idéalement représentée, mais plutôt transmis pour être approfondi, c'est-à-dire toujours «plus profond en soi... (dans) le sens d'une expérience (du vécu personnel) ne s'adressant pas au seul intellect, mais à la personne tout entière » (Cheng, 1997, p. 34) en vue de sa "disparition » en soi pour l'émergence future d'un dispositif efficient, au moment juste.

L'efficacité est le seul but de cette action occidentale. C'est d'ailleurs à travers l'atteinte d'un niveau d'efficacité attendu que cette action sera jugée. Son aptitude propre à mettre en œuvre les moyens prévus, à contrôler (évincer ?) les circonstances perturbatrices, à fournir l'effort nécessaire, conduira l'homme d'action à maîtriser les éléments pour atteindre le but spécifié. La vision chinoise, on l'a compris, s'engage moins dans un 
rapport de moyen/fin que dans un rapport de conditions/conséquences. Il s'agit moins d'atteindre le but que de faire évoluer les conditions à son profit. Il y a moins ambition d'atteindre un idéal, que d'exploiter les dispositions afin de "faire croittre progressivement l'effet» (Jullien, 2005, p. 75). Si nous avons compris l'importance des facteurs porteurs, nous pourrions cependant être tentés, en qualité d'occidentaux, de percevoir cette efficacité uniquement à travers les valeurs propres de la personne, les circonstances n'étant que hasard. Mais comme le questionne Dubet (2004, p. 20) «dans quelle mesure peut-on considérer que les individus sont responsables de leurs talents, de leur courage et de leur volonté»? Là où l'Occident voit un homme courageux (ou lâche), qualité qui semble dispositionnelle, attachée à l'homme dès sa naissance, la Chine perçoit une caractéristique situationnelle. En bref, à l'efficacité occidentale, dépendante de l'action humaine et attachée à un but, l'efficience chinoise ne s'intéresse qu'aux conditions et au rendement (Jullien, 2005, p. 75).

\section{Mise en perspective}

Comme nous le précisions en introduction, notre but dans ce travail n'était ni de justifier une nouvelle pratique, ni de critiquer les méthodes actuelles, mais plutôt de comprendre une approche différente à travers deux grilles de lecture. Arrivés maintenant à la dernière partie, nous constatons que si c'est notre pratique qui nous a entraînés dans le travail de rédaction, l'analyse des différences et les lectures qu'en ont permisla notion de bricolage et la pensée chinoise nous mènent à réfléchir aux enseignements et aux questions que ces détours peuvent amener dans une conception du coaching. L'enseignement général de ce travail, mais qui pourrait être tout autant philosophique et éthique, est certainement celui du « regard décalé » : prendre un point de vue différent, pour mieux comprendre un problème. Que nous dit cet « outil» central des pratiques de coaching? Que nos convictions, nos préjugés, nos certitudes ou encore nos pratiques quotidiennes peuvent nous conduire à créer un cadre de référence rigide au point de bloquer notre aptitude à penser « hors cadre "pour trouver une solution. En appliquant ceci à notre réflexion, nous comprenons alors dans un premier temps, qu'une pratique qui peut être jugée de «bricolage » à l'aune de la rationalité et du modèle cartésien, et c'était parfois notre jugement autocritique, peut non seulement être comprise par un regard décalé, mais devenir à son tour un référentiel d'analyse. En effet, si le détour par la Chine a montré une certaine rationalité, il permet également de questionner le coaching tel que présenté et pratiqué en Occident. Ainsi, comment une pratique qui se donne pour objet principal le changement, le développement des potentiels, peut-elle proposer une démarche fondamentalement basée sur les mêmes schémas de fonctionnement que ses clients, les mêmes archétypes, sous-tendus par le principe de modélisation : diagnostic, définition d'un but idéal, planification, utilisation d'outils adaptés? Peut-on voir différemment, si l'on regarde de la même manière?

Si l'on parle de manière, une seconde question survient d'elle-même: qu'est-ce qu'apprendre ou qu'est-ce que changer ? Il faudra alors répondre au quoi et au comment. Dans leur revue de littérature, Kampa-Kokesch et Anderson (2001) synthétisent le recours à cette pratique de coaching par les managers par un recours à l'extérieur pour " recevoir ce dont ils manquent» (p. 209). Le quoi serait donc un vide à combler, en vue d'une amélioration de la performance des managers, à travers une acquisition de connaissances, de méthodes et technique, que le contrat porte sur le développement 
personnel, relationnel, ou managérial. Si tous les coachs ne se retrouvent probablement pas dans cette affirmation, il est cependant clair que les contrats ont bien tendance à montrer en quoi l'action va réduire l'écart (le vide) existant avec le but envisagé. De son côté, la pensée chinoise, à la question du quoi, propose avant tout d'apprendre à faire de soi un être humain : " la grande question de l'apprendre consiste avant tout d'apprendre à être humain» (Cheng, 1997, p. 67). Il est donc plus fait appel ici aux potentiels originels des personnes qu'à leurs capacités d'acquisition. C'est à partir de ce socle personnel, et non pas des méthodes, que l'agir pourra prendre sa place. Il en sera de même pour le comment. C'est dans une attitude d'apprendre, plus que par un apprentissage, que le coach et le manager avanceront vers une évolution : «il ne s'agit pas tant d'une démarche intellectuelle que d'une expérience de vie» (p. 65). L'apprendre sera vécu comme un partage où chacun apporte ses éléments. Nous pouvons retrouver ce type d'approche dans la pensée occidentale. Ainsi, Rogers (1998, p. 197) considère que l'apprendre "authentique " pourra avoir lieu si l'enseignant développe « une relation personnelle avec ses étudiants (ainsi) qu'un climat dans sa classe tels que ces tendances naturelles (de l'élève) arrivent à leur pleine maturité ». Si l'on ne considérait que l'ensemble des travaux de Rogers et leurs validations, qui viennent rassurer notre démarche cartésienne, ne pourrait-on pas concevoir une approche du coaching moins centrée sur les moyens et leur acquisition que sur la relation, la personne et son inscription dans une situation? Moins centrée sur le développement des potentiels pour un agir original - moi qui agis sur la situation -, mais plus sur l'éclosion des potentiels vers un agir originel, en se rapprochant de la personne que je suis réellement - soi qui trouve sa place et s'inscrit dans le monde.

Déjà entendons-nous nos détracteurs, coachs ou managers: "qu'en sera-t-il alors de la performance » ?Et c'est peut-être là finalement que les craintes sont les plus grandes, mais là aussi qu'un autre point de vue peut apporter un regard neuf sur une pratique. Oui, la performance est importante, oui la performance est bien la raison pour laquelle une entreprise paye un prestataire tel que le coach et oui, enfin, l'objet de l'intervention est bien de permettre aux managers, à terme, de gérer de façon performante les situations qu'ils rencontrent. Cependant, cette performance sera-t-elle valide uniquement parce qu'il y aura acquis de connaissances, de savoir ou de savoir-faire, réduisant la prestation de coaching à une simple logique client-fournisseur ou, à l'opposé, doit-elle se plonger dans une relation de co-investissement, de partage et de transformation, des compétences, des pratiques ou des analyses? Nous pensons que le coach se doit de passer de l'estrade au plancher, d'accepter de perdre l'autorité des savoirs détenus pour cofonder l'évolution du manager dans la situation problème évoquée. Cela veut-il dire perdre son rôle de coach? Il ne nous semble pas, car si l'objectif de la prestation de coaching est de permettre au manager de gérer au mieux une situation, la question sera bien de savoir s'il vaut mieux l'aider à trouver les moyens pour atteindre son but ou à l'inverse l'accompagner à comprendre et naviguer sur les facteurs porteurs. Doit-on chercher un résultat visible et immédiat (pour le manager et pour le coach d'ailleurs) ou veut-on savoir détecter les signaux faibles et transformer, de façon plus discrète mais à long terme, les conditions nécessaires telles que l'efficacité en résulte? Peut-être un peu des deux... Cependant, que ce soit l'un ou l'autre, il est du ressort du coach de faire le nécessaire pour que son intervention entre bien dans le cadre d'un « mieux vivre » pour le manager. 


\section{BIBLIOGRAPHIE}

Baker T., Miner A., et Eesley D., (2003), «Improvising Firms: Bricolage, Account Giving, and Improvisational Competency in the Founding Process », Research Policy (32), pp. 255-276.

Ben-Ari M., (1999), « Bricolage forever! »,pp. 53-57 In: 11th Workshop of the Psychology of Programming Interest Group. Leeds (UK), September 10, 2005 ; http: www.ppig.org/papers/11 thbenari.pdf.

Cheng A., (1997), Histoire de la pensée chinoise, Paris, Seuil.

Conference, I. C. F. (2000), International Executive Coaching Summit: a Collaborative Effort to Distinguish the Profession, http://www.coachdederation.org/ecsummit-1999 .htm\#b. [2000, July 20].

Confucius, (1981), Les entretiens de Confucius (A. Cheng, Trans.), Paris, Éditions du Seuil.

Délivré F., (2002), Le métier de coach. Paris, Les Éditions d'Organisation.

Dubet F., (2004), « Critique de la performance comme modèle de justice» pp. 15-27 in :

B. Heilbrunn (Ed.), La performance : une nouvelle idéologie ?, Paris, La Découverte.

Duymedjian R. et Rulling C.-C., (2005), Bricolage and Situated Performing in Organizations, Paper presented at the 21 st EGOS colloquium: Unlocking Organizations, Berlin.

Gautier B. Vervisch M.-O., (2005), Le manager-coach (2e éd.), Paris, Dunod.

Hévin B. \& Turner J., (2002), Manuel de Coaching, Paris, Dunod.

Jullien F., (1996), Traité de l'efficacité, Paris, Grasset.

Jullien F., (2005), Conférence sur l'efficacité, Paris, P.U.F.

Kamenarovic I. P., (2001), Le conflit : perception chinoise et occidentale, Paris, Éditions du Cerf.

Kamenarovic I. P., (2005), Agir, non-agir en Chine et en Occident, Paris, Éditions du Cerf.

Kampa-Kokesh S., \& Anderson M. Z., (2001), « Executive Coaching: a Comprehensive Review of the Litterature», Consulting Psychology Journal, 53 (4), pp. 205-228.

Kilburg R. R., (2000), Executive Coaching: Developping Managerial Wisdom in a World of Chaos, Whashington, DC: American Psychological Association.

Lévi-Strauss C., (1962), La pensée sauvage, Paris, Plon.

Perrenoud P., (1983), « La pratique pédagogique entre improvisation réglée et le bricolage : essai sur les effets indirects de la recherche en éducation ", Éducation et Recherche (2), pp. 198-212.

Rogers C., (1998), Le développement de la personne, Paris, Dunod.

Weick K. E., (1998), « Introductory essay: Improvisation as a Mindset for Organizational Analysis », Organization Science, 9 (5), pp. 543-555.

Whitmore J., (2003), Le guide du coaching, Paris, Maxima.

Winum P. C., (2005), Effectiveness of a High-Potential African American Executive: the Anatomy of a Coaching Engagement, Consulting Psychology Journal, 57 (1), pp. 71-89. 


\section{NOTES}

1. André Gide, Les Nourritures terrestres (1917-1936).

2. Biofeedback: utilisation de paramètres psychophysiologiques à des fins d'apprentissage d'auto-régulation.

3. Carl Rogers, Le développement de la personne, Dunod, 1998, p. 189.

4. Délivré, Le métier de coach, Les Éditions d'organisation, 2002.

\section{RÉSUMÉS}

Cet article se propose de présenter une pratique de coaching spécifique et d'en fournir divers moyens d'analyse. Après la narration d'une expérience concrète, il définira dans un premier temps les grands éléments qui la différencient des approches plus conventionnelles. Un détour par la science du concret et par la pensée chinoise donnera ensuite la possibilité d'une lecture plus générale de cette approche. L'objet de ce travail n'est nullement de définir ou de justifier un nouveau modèle, mais bien plus de partager et d'ouvrir de nouvelles perspectives de recherche et de développement de pratiques.

This article seeks to present a specific type of coaching and different means to analyse. It begins by describing a real experience and then defines the major elements that differentiate this experience from the more conventional approaches. Borrowing from the Science of the Concrete and Chinese thought will help us provide a more general analysis of the approach. The work herein does not seek to define or justify a new model, but rather to open up and share new perspectives in research and development of practices in coaching.

\section{AUTEUR}

\section{DOMINIQUE STEILER}

Dominique Steiler est professeur associé à Grenoble, École de Management, où il dirige le Centre Développement Personnel et Managérial. Son domaine de recherche porte sur le développement personnel, la gestion du stress et le coaching. Il est par ailleurs coach auprès de managers et de dirigeants. Mail : dominique.steiler@grenoble-em.com 